\title{
Further studies on the treatment of ocular onchocerciasis with diethylcarbamazine and suramin
}

\author{
J. ANDERSON AND H. FUGLSANG \\ From the London School of Hygiene and Tropical Medicine
}

SUMMARY The purpose of the present study was to determine whether or not the elimination of microfilariae by diethylcarbamazine citrate (DEC) would reduce the side effects of a subsequent course of suramin and thus make it more acceptable and safer in the treatment of onchocerciasis. Fifty-four heavily infected patients with ocular onchocerciasis were treated with an initial 2-week course of DEC, in which the dose was increased slowly from $25 \mathrm{mg}$ on the first day to 150 to $200 \mathrm{mg}$ twice daily on days 8 to 14 . Betamethasone $1.5 \mathrm{mg}$ was given twice daily 2 days before DEC administration, and during the days of increasing DEC dosage, after which it was tailed off. This course was followed by 3 to 5 weekly injections of suramin. It was found possible to rid these heavily infected patients of most of their microfilariae during the initial course without intolerable side effects. The subsequent suramin course was in general well accepted except in 1 case, where there was a serious aggravation of anterior uveitis. The later development of retinal pigment atrophy in 3 patients, of optic atrophy in 3 others, and of both retinal and optic atrophy in 1 patient is discussed.

The effects of this regimen on eye lesions and visual acuity at the final examinations (1 to 4 years) compared favourably with those from our previous trial of suramin given alone or followed by DEC, and they can be summarised as follows:

\begin{tabular}{|c|c|c|c|c|c|}
\hline \multirow{2}{*}{ Lesions } & \multirow{2}{*}{$\begin{array}{l}\text { Before } \\
\text { treatment }\end{array}$} & \multicolumn{2}{|c|}{ After treatment } & \multirow[b]{2}{*}{ Worse } & \multirow[b]{2}{*}{$\begin{array}{l}\text { New } \\
\text { lesions }\end{array}$} \\
\hline & & Better & Same & & \\
\hline Sclerosing keratitis & 18 & 15 & 3 & 0 & 0 \\
\hline Iritis & 30 & 24 & 6 & 0 & 0 \\
\hline Glaucoma & 5 & 0 & 2 & 3 & 0 \\
\hline Choroidoretinitis & 25 & 0 & 20 & 5 & 4 \\
\hline Optic atrophy & 19 & 0 & 19 & 0 & 4 \\
\hline Optic nerve congestio & n 6 & 0 & 0 & 6 & 0 \\
\hline Visual acuity (eyes) & 108 & 13 & 73 & 22 & - \\
\hline
\end{tabular}

It is generally accepted (World Health Organisation, 1974) that ocular disease in onchocerciasis is caused by microfilariae of Onchocerca volvulus in the ocular tissues. However, it is not clear how much disease is caused by the presence of living microfilariae and how much by their death and dissolution (Anderson and Fuglsang, 1977). Host reactions to microfilariae vary not only between different areas and between different individuals, but also within the same individual at different stages of the infection (Bryceson, 1976; Bartlett et al., in press). Variations might therefore be expected in response to the administration of macro- and microfilaricidal drugs.

Unpredictable and dangerous general reactions to both suramin (Nelson, 1955; Satti and Kirk, 1957; Budden, 1959; Anderson et al., 1976a) and

Address for reprints: Dr J. Anderson, 77 Pennard Road, London W12 8DW

diethylcarbamazine citrate (DEC) (Oomen, 1969; Fuglsang and Anderson, 1974a; Bryceson et al., 1977) have been reported, indicating the need for caution in the management of heavily infected patients. In the skin local reactions to the destruction of microfilariae by DEC and/or suramin can result in the formation of microabscesses (Connor et al., 1970; Fuglsang and Anderson, 1974b). Similarly severe inflammatory reactions might therefore be expected in the eye after the administration of microfilaricidal drugs, and a major problem in the treatment of ocular onchocerciasis is the avoidance of reactions that may cause irreparable damage to the delicate ocular tissues.

In a previous paper (Anderson et al., 1976b) we have described in some detail the effects of a single 10 to 14-day course of DEC on heavily infected eyes, most of which already had 1 or more lesions due to onchocerciasis. The effects of a subsequent 
weekly 'suppressive' dose of DEC were also described. Despite the administration of a corticosteroid (Markell and Turner, 1957) 1 day before and on the first 5 to 10 days of DEC, the drug proved very difficult to administer to these patients with severe ocular involvement. There was always an initial aggravation of inflammatory reactions during the first 3 days of treatment, and after a single course any beneficial effect lasted only a matter of months. With a subsequent weekly dose it was possible to keep the number of microfilariae in the skin and eye at a low level. In this way it could halt or cure lesions of the anterior segment such as sclerosing keratitis and iritis, but glaucoma was, in general, unaffected by DEC administration and usually progressed to serious loss of vision or blindness. Choroidoretinal lesions sometimes progressed despite or even because of treatment, and it could not be ruled out that the intensive DEC course caused some congestion of the optic nerve head. Once started, disease of the optic nerve almost invariably progressed to serious loss of vision or blindness. It is likely that the DEC dosage was too high and concentrated, and that the body could not easily dispose of the enormous numbers of newly-killed microfilariae. Another possibility was that the corticosteroid administration was inadequate to prevent the ensuing inflammatory reactions. Further studies are needed to identify therapeutic regimens which, by balancing correctly the microfilaricidal and anti-inflammatory effects, avoid damaging ocular reactions.

In heavily-infected patients, when very severe reactions to DEC are expected, Duke and Anderson (1972) suggested that treatment should begin with a course of suramin. The microfilaricidal effect of suramin would eliminate the source of macrofilariae, which would then gradually disappear from the skin and eye. Its microfilaricidal properties, much less than those of DEC, might not be so immediately damaging. We therefore gave suramin to 100 heavily-infected patients (Anderson et al., 1976a). It was found that it caused serious and unacceptable general reactions, not all of which could necessarily be attributed to its parasiticidal effects (Fuglsang and Anderson, 1974b). Localised inflammatory reactions round the microfilariae also occurred in the skin and eye. These reactions were most pronounced after the third or fourth injections, when blood concentrations of suramin were starting to reach filaricidal levels (Duke, 1968a). At the same time there was aggravation of sclerosing keratitis, and sometimes development or serious aggravation of anterior uveitis, resulting in the formation of posterior synechiae. Simultaneous congestion of the optic nerve head was suggested on clinical examination, and some cases of optic atrophy developed after treatment. Angiographic studies were not performed. Despite these reactions the effects of suramin on anterior segment ocular lesions were in many cases more beneficial and of much longer duration than those of a single course of DEC. As for lesions of the posterior segment, the best results to be hoped for was an arrest of the further development of such lesions.

The question remained whether or not some of the serious and unacceptable side effects of suramin could have been prevented (1) by prior elimination of the microfilariae by DEC, or (2) by adequate administration of corticosteroid drugs, and the main aim of the present study was to test the former hypothesis. Fifty-four heavily-infected patients were therefore given a 2-week course of DEC under cover of betamethasone, and their response to this and a subsequent course of suramin was followed up over 1 to 4 years.

\section{Materials and methods}

The 54 patients, 45 males and 9 females aged between 8 and 50 years, all lived in the area of Touboro, a village in the Sudan savanna of north Cameroon where the previously-reported studies also took place. The patients presented themselves at our field clinic. Forty-seven among them already had onchocercal eye lesions, while 7 were considered to be at risk of developing lesions because of heavy invasion of microfilariae in and around the eye (Fuglsang and Anderson, 1977). All were in fair to good general condition, able to perform a normal day's work in the field.

The aim of the initial DEC course was to reduce the microfilarial density in the skin to near zero by giving the drug at the following dosage (mg):

\begin{tabular}{lllllllllll}
\hline Day & 1 & 2 & 3 & 4 & 5 & 6 & 7 & 8 & 9 & $10-14$ \\
\hline Morning & 0 & 25 & 50 & 50 & 100 & 100 & 150 & 150 & 200 & 200 \\
Evening & 25 & 50 & 50 & 100 & 100 & 150 & 150 & 200 & 200 & 200 \\
\hline
\end{tabular}

Patients under the age of 10 received half dosage. Ten patients (Group I) also received $200 \mathrm{mg}$ DEC per week for 12 weeks after the initial DEC course but before suramin. Betamethasone was given routinely in a dosage of $1.5 \mathrm{mg}$ morning and evening on the 2 days before the start of the DEC therapy and during the days of increasing DEC dosage, after which it was tailed off. These drugs were always given after a light meal.

An average adult received a full gramme of suramin at each weekly injection, but young, small, or thin patients (less than $50 \mathrm{~kg}$ ) received an amount 
corresponding to their weight. No test dose was given, but all the injections were given very slowly, and in Groups I and II the patients only received a half dose at the initial injection. Before each injection the urine was tested for albumin by the Albustix method.

The patients were grouped according to the drug schedules received and the length of follow-up:

\begin{tabular}{lllll}
\hline \multicolumn{5}{c}{ Diethylcarbamazine } \\
Group & No. seen & Single course & Weekly dose & Suramin \\
\hline$I$ & 10 & 2 weeks & 12 weeks & $4.5 \mathrm{~g}$ \\
II & 12 & 2 weeks & nil & $4.5 \mathrm{~g}$ \\
III & 26 & 2 weeks & nil & $4.0 \mathrm{~g}$ \\
IV & 6 & 2 weeks & nil & $3.0 \mathrm{~g}$ \\
\hline
\end{tabular}

A general physical examination and detailed eye examination were carried out on the numbers of persons indicated before treatment and at the following intervals:

\begin{tabular}{lllllll}
\hline \multicolumn{7}{c}{ Months after treatment } \\
Group & Before treatment & 9 & 12 & 24 & 36 & 48 \\
\hline I & 10 & - & 7 & 7 & 8 & - \\
II & 12 & - & 9 & 10 & 9 & 7 \\
III & 26 & 26 & - & 9 & - & - \\
IV & 6 & - & 6 & - & - & - \\
\hline
\end{tabular}

Additional eye examinations were carried out during the DEC and suramin courses, and in some cases up to 2 weeks after the last suramin injection. Skin snips were taken with a corneoscleral punch from the left side of the body at the outer canthus, shoulder, and buttock. The snips were left for 4 hours in 2 drops of normal saline in separate wells of a microtitration plate. After removal of the snips with a pin, the saline from each well was transferred by pipette to separate slides, and the microfilariae which had emerged were counted under a microscope at low power.

The eyes were examined as described by Anderson et al. (1974). In brief, vision was tested using an illiterate $\mathrm{E}$ chart, and blindness was defined as the inability to count fingers accurately at $3 \mathrm{~m}$ with the better eye. A Haag-Streit 900 slit lamp was used to examine the anterior segments, and direct and binocular indirect ophthalmoscopy for the posterior segments. The maximum number of microfilariae seen in either eye in the cornea, anterior chamber, before or after positioning (Anderson and Fuglsang, 1973), and posterior to the lens was recorded at each examination. Measurements of the intraocular pressure were made in as many patients as was possible before treatment, using the applanation method, but subsequent readings were obtained only from those in whom the original levels had been abnormally high or when a raised pressure was suspected on clinical grounds. Visual fields were tested using a simple confrontation test (Bird et al., 1976). All the above examinations were made by the same observers throughout.

\section{Results}

GENERAL ACCEPTABILITY AND SIDE EFFECTS OF THE DRUG

$D E C$. Most of the patients adhered to the course, but not without some persuasion, and some patients omitted 1 or 2 doses, especially on the third to fifth day of DEC treatment. Their main complaint was 'head turning'. For the same reason a few patients were not able to take as much as $200 \mathrm{mg}$ twice daily. The course was therefore adjusted slightly according to individual response. They all experienced severe tenderness of regional lymph nodes, often aggravated when the betamethasone was tailed off. One patient developed oedema of a leg, another oedema of the genitalia, and a third of 1 breast. These swellings subsided gradually, but sometimes not until 1 to 3 weeks after treatment.

Suramin. Two of the 54 patients experienced nausea and abdominal discomfort immediately after the first injections, but these symptoms lasted only for a few minutes and did not recur at subsequent injections. Pain in the feet sometimes occurred on the day following an injection, but it was not severe and subsided over the next 2 to 3 days. General malaise was common during the week following the last 2 injections. However, the patients continued to move around, though they were not fit for heavy physical work. Five of the group IV patients received only $3 \mathrm{~g}$ because of absenteeism, but in the sixth the drug was discontinued after the third injection because of aggravation of iritis (see below). Slight transient albuminuria occurred in most patients, but injections were not withheld on this account.

\section{MICROFILARIAE IN THE SKIN}

Table 1 shows the changes in the mean concentration of microfilariae per snip (average of the 3 snips) in the different groups. The DEC course reduced the microfilarial load to about $5 \%$ of its original value, and the suramin course kept it down to about $10 \%$ for as long as 4 years despite the continuous exposure to re-infection.

MICROFILARIAE IN THE EYE

The patients in groups I and II responded similarly 
Table 1 Showing the pretreatment arithmetic mean concentration of microfilariae per snip (average of 3 skin snips), and the post-treatment mean concentration expressed as a percentage of the pretreatment value

\begin{tabular}{llllllll}
\hline & $\begin{array}{l}\text { Pre- } \\
\text { treatment } \\
\text { concen- } \\
\text { tration }\end{array}$ & $\begin{array}{l}\text { Post- } \\
\text { Dec }\end{array}$ & $\begin{array}{l}\text { Post-suramin }(\%) \\
9 \text { months }\end{array}$ & $\begin{array}{l}\text { 1 year } \\
\text { Group }\end{array}$ & 2 years & 3 years & 4 years \\
\hline I & 132 & 3 & 4 & 5 & 6 & \\
II & 84 & 5 & 8 & 13 & 8 & 10 \\
III & 108 & & 9 & & 5 & & \\
IV & 119 & & & 23 & & \\
\hline
\end{tabular}

with respect to microfilariae in the eye, and they are therefore described together.

Microfilariae in the cornea (MFC) were present before treatment in all 22 patients in groups I and II combined, and more than 100 MFC were estimated in half these patients. At the follow-ups held after 1,2 , and 3 years the prevalence was reduced to 44 , 6 , and $24 \%$ respectively, and the intensity of MFC was much reduced. Thus at year 1 the maximum number of MFC was 2 to 5 , at year 2 it was 1 only, but at year 3 over 20 MFC were seen in 1 patient, though the 3 other positive cases at this stage each showed 1 MFC only. Living microfilariae, present in all patients before treatment, were seen in 4 patients at year 1 , in none at year 2 , and in 2 at year 3 .

Before treatment 11 of the 26 patients in group III showed more than $100 \mathrm{MFC}$, and the prevalence was again $100 \%$. At 9 months and 2 years the prevalence was 32 and $44 \%$ respectively, but the intensity was again much reduced. In group IV at 1 year the prevalence of MFC had fallen from 6 out of 6 to 3 out of 6 . Two of the positive cases now showed only 1 MFC, while about 10 were present in the third.

Microfilariae in the anterior chamber (MFAC) were seen in 18 of 22 patients $(82 \%)$ before treatment in groups I and II combined, and in 4 of them more than 50 MFAC were estimated. At years 1, 2 , and 3 the prevalences were 38,35 , and $41 \%$ respectively, but the maximum number seen was only 2 to 5 .

Before treatment the prevalence of MFAC in group III was $77 \%$. At 9 months it was $28 \%$ and at 2 years $56 \%$. At 9 months the maximum number counted per anterior chamber was 2 to 5 , but at 2 years about 10 were present in 1 of the positive cases. In group IV the pretreatment prevalence was 6 out of 6 , but at 1 year it was reduced to 2 out of 6 , with a maximum of 2 to 5 MFAC.

Microfilariae on the posterior lens surface and/or in the vitreous. Before treatment and at the follow-ups after 1,2 , and 3 years this sign was present in 23 , 31,24 , and $18 \%$ respectively in groups I and II combined. All these microfilariae were dead at each examination. In group III the prevalence was 23,24 , and $11 \%$ at the pretreatment, 9-month, and 2-year examinations respectively. In group IV a living microfilaria was seen before treatment in each vitreous humour of 1 patient. After the course of DEC dead microfilariae were present in the vitreous in this same patient.

OCULAR LESIONS-RESPONSE TO THE INITIAL COURSE OF DEC

The immediate response to DEC therapy has been described in detail in a previous paper (Anderson et al., 1976b). Despite the more generous administration of betamethasone in the present study exacerbations of ocular inflammation occurred in the form of watering, photophobia, bulbar and circumcorneal hyperaemia, the formation of minute globular infiltrates at or near the limbus, and of fluffy punctate opacities. An initial migration of microfilariae into the cornea also occurred. However, these effects were not severe and caused little discomfort.

Sclerosing keratitis was present in 18 patients before treatment in all the groups combined. A further 7 showed haziness of the nasal and/or temporal cornea, associated with massive corneal parasitisation. During the course of DEC there was a slight increase in overall haze in some cases, but this was of short duration. By contrast many of the established cases of sclerosing keratitis showed an increasing attenuation in the extent and severity of corneal involvement.

Iritis. Signs of past or present iritis were seen in 30 patients before treatment. Heavy flares with fresh-looking keratic precipitates were present in 5 patients, in 3 of whom extensive posterior synechiae had already formed. Fine flares with circulating cells were present in a further 25 patients, of whom 6 showed posterior synechiae. During the course of DEC there was little or no increase in the severity of iritis, and by the end of the course there was a considerable improvement in some cases. This improvement was particularly marked in 1 patient at the end of the 12-week 'suppressive' course of DEC. Before treatment there were massive keratic precipitates, heavy flares, and extensive posterior synechiae in both eyes, while after DEC treatment all the keratic precipitates had been absorbed and only fine flares were present, though the extent of posterior synechiae remained unchanged.

Early in the DEC course large numbers of microfilariae became attached to keratic precipitates 
in 2 patients, while in a third microfilariae attached themselves to a small lump of exudate.

Glaucoma. Five of the cases of iritis were associated with glaucoma, and the intraocular pressures were largely unaffected by the course of DEC.

Choroidoretinitis. Fundus lesions ranging from very mild temporal atrophy of the pigment epithelium to gross lesions affecting much of the posterior pole were diagnosed before treatment in 25 patients in all groups combined. No significant changes were noticed in their appearance during the course of DEC, and changes were not seen in previously healthy fundi.

Optic disc. Optic atrophy, usually associated with fundus lesions, was already established before treatment in 19 patients. More active disease with congestion and swelling of the optic nerve head was seen in a further 6 patients. Extensive visual field loss was found in all these patients. Early changes with DEC treatment were not seen in the congested discs, and previously normal optic discs remained apparently normal.

In summary it may be said that the anterior segments were in general improved at the end of the DEC/betamethasone course, while the posterior segments were unchanged.

OCULAR LESIONS--RESPONSE TO THE COURSE OF SURAMIN FOLLOWING DEC

The immediate response to treatment with suramin alone has been described in detail in a previous paper (Anderson et al., 1976a).

Sclerosing keratitis. The lessening of the extent and density of sclerosing keratitis, which was seen during the course of DEC, continued during the suramin course and at subsequent follow-ups. Of the original 18 cases none was worse at the final examination, 3 were more or less unchanged, 8 showed some corneal clearing, and the remaining 7 were virtually normal. In several instances there was undoubted migration of pigment out of the cornea, together with a gradual reduction in the extent of corneal vascularisation, subsequent to the dissolution of the corneal microfilariae. The heavily parasitised corneas in which nasal and/or temporal haze only was present all became absolutely clear.

Iritis. During the course of suramin a slight increase in the amount of flare was noted in 2 patients. In another a heavy flare was seen 1 week after the third injection, so that further injections were not given to this patient. No increase of iritis was noted in the other 27 patients who showed pretreatment signs, and no case developed de novo. Of the original 30 cases with some degree of iritis 6 were more or less unchanged at the final examination,
8 were improved though still showing some signs of iritis, and the remaining 16 had cleared. All 5 cases which showed heavy flares and fresh keratic precipitates before treatment were markedly improved, showing fine flares only and no keratic precipitates at the final examination. Their posterior synechiae were unchanged.

Glaucoma cases showed no improvement throughout the period of observations.

Choroidoretinitis. Among the 25 patients who showed some degree of choroidoretinal atrophy before treatment the changes were more marked in 5 at the final examination, while they appeared unchanged in 20 . Four patients with previously healthy fundi showed mild temporal changes at the last examination.

Optic disc. There were no changes in the 19 patients with established optic atrophy. The 6 patients who showed congestion and swelling before treatment had all developed optic atrophy by the last examination. Four other patients whose optic discs were considered normal before treatment developed optic atrophy, although a congestive phase was not seen in them.

\section{VISUAL ACUITY}

For the majority of eyes, 73 out of 108, central visual acuity was the same at the end of observations as at the beginning. An improvement of 2 steps or more on the $E$ chart was recorded in 13 eyes, and this was attributed to corneal clearing in 8 , to a lessening of iritis in 3 , while no obvious reason was found in the other 2. A deterioration of 2 steps or more on the chart was recorded in the remaining 22 eyes. In 6 this was attributed to glaucoma, in 7 to progress in posterior segment disease, and in 2 to increased cataract, while in the remaining 7 no obvious reason was found.

Two patients in group III became blind during the observations. One was a boy of 13 whose vision before treatment was $6 / 9$ (right) and no perception of light (left). Both eyes showed optic atrophy and massive choroidoretinal atrophy involving the entire posterior pole. The physical signs in the right eye showed no major changes at the last examination, but central visual acuity had been lost. The other patient, a boy of 12 , had a pretreatment vision of 6/36 and no perception of light. He presented with a very large group of nodules behind 1 ear, and his eyes were grossly parasitised, with an estimated 1000 to 2000 microfilariae in each cornea. Lid swelling and ocular inflammation made intraocular pressure recording very difficult. At later examinations, when the microfilariae had gone, corneal epithelial oedema was seen in both eyes, and the optic discs were deeply cupped. His vision 
at the final examination was no perception of light with either eye.

No other individual eyes became blind during the observations, and in 4 other patients 1 eye improved from recognition of hand movements to $6 / 36,6 / 36,6 / 18$, and $6 / 6$ respectively. In the 2 former cases there had been corneal clearing and improvement in signs of iritis, but no change in physical signs could reasonably account for the better visions in the 2 latter cases. The fellow eyes in these 4 patients had good central vision throughout the period of the trial.

\section{Discussion}

In general the DEC regimen used in the present study, starting with $25 \mathrm{mg}$ instead of $50 \mathrm{mg}$ and increasing slowly over 7 days instead of 3 days, was more acceptable and provoked less severe side effects than the previously reported regimen. Undoubtedly the betamethasone cover $(1.5 \mathrm{mg}$ twice daily), starting 2 days instead of 1 day prior to DEC administration and continuing for a longer period, helped to reduce the side effects, though they were not prevented altogether. We have subsequently given a similar DEC course to a very heavily-infected patient, but he received betamethasone $1.5 \mathrm{mg}$ three times a day 3 days prior to and during the first 7 days of DEC therapy. He did not complain of any side effects apart from slight lymph node tenderness, and the course resulted in a substantial reduction of his microfilarial load. More studies are necessary to balance the betamethasone dose against the DEC dose, not only in these heavilyinfected patients but also in more lightly-infected ones.

Many textbooks advocate giving DEC three times daily, but the advantage of this regimen over a twice-daily dose is doubtful, not only on clinical grounds but also in view of the findings of Lubran (1950) and Bangham (1955) on DEC absorption and excretion. Further studies on the pharmacodynamics of DEC are long overdue and of special interest now that transdermal and other drug delivery systems are making it possible to maintain more constant drug levels (Jones et al., in preparation).

Our experiences to date indicate that with a correctly balanced and supervised DEC/betamethasone course it is possible to rid even the most heavily-infected patients of microfilariae without unacceptable side effects. The subsequent suramin course was also tolerated better than the previously reported suramin trial, in which microfilariae were not first eliminated by DEC. Admittedly the number of patients treated in the present study was only 54 , but even so there were fewer alarming side effects such as occurred when 100 patients received suramin alone ( 2 deaths, 1 exfoliative dermatitis, 1 stomatitis, 3 severe cases of iritis). Similarly the general malaise was less pronounced and there were no cases with papular skin eruptions. However, the aggravation of iritis in 1 case must not be forgotten. No marked aggravation of anterior uveal irritation was seen in the 10 patients who had received suppressive DEC between the initial DEC course and the suramin course. It is therefore possible that this presuramin regimen is the best, since it allows time for most inflammatory reactions to quieten. On the other hand, from the patient's point of view the most practical and least time-consuming course would be the simultaneous administration of DEC/ betamethasone and suramin. This would seem worth a trial, but those investigators who have seen the worst side effects of both drugs given separately might well be reluctant to embark on such a trial before a better understanding of the mode of action of both drugs is reached and before the toxicity of suramin is clarified (Gibson et al., 1977).

It might even be possible to make acceptable a short suramin course consisting of daily injections. Duke (1968a), in his extensive studies on suramin, found that such a 'rapid' course of daily injections to a total of $2.7 \mathrm{~g}$ had satisfactory macrofilaricidal effect in the Cameroon rain forest, but 'that it was poorly tolerated and could not be recommended for general use'. In the present study, judged by the decrease in the microfilarial load in the skin and eye (Duke, 1968b), even $3 \mathrm{~g}$ of suramin given weekly had a considerable macrofilaricidal effect; $4 \mathrm{~g}$ failed to give completely satisfactory results in only 2 of 26 patients, and $4.5 \mathrm{~g}$ produced a good macrofilaricidal effect in all cases. It is generally agreed that a high suramin dosage is toxic. It would therefore seem essential to establish the minimum macrofilaricidal blood level of suramin and for how long it has to be maintained. There may well be variations both between individuals and between areas (Hawking, 1940).

Our previous conclusions on the use of DEC and suramin in ocular onchocerciasis were rather pessimistic. From the effects on eye lesions of the present regimen we are more optimistic, especially with respect to lesions of the anterior segment, excluding glaucoma. Glaucoma is a serious problem in onchocerciasis, and it is hoped that those ophthalmologists who have used both surgical intervention and antifilarial treatment will soon publish their experiences. In the posterior segment the microfilarial load cannot be assessed clinically as in the anterior segment, and early inflammatory changes are probably often overlooked in routine 
clinical examinations, as indicated by studies using fundus angiography (Metge et al., 1974; Trojan, 1975; Bird et al., 1976). During healing these early lesions would necessarily leave some residual changes, and it would be impossible to evaluate by clinical means whether or not this healing process was aggravated by the treatment as such. Fundus angiography during treatment might help to answer that question.

In the present study the 4 new clinical cases of choroidoretinitis all consisted of mild atrophy of the retinal pigment epithelium temporal to the macula, and they may well have been the end result of processes which were not detected at the pretreatment examination. Unfortunately, many of the patients in this study could not be followed up closely during the weeks following the suramin course, and there was no 3-month follow-up. During this period the 4 patients who developed optic atrophy de novo might have shown signs of active optic nerve disease. The question whether or not treatment can aggravate inflammation of the optic nerve can probably also be solved only by angiographic studies during treatment and/or by more accurate visual field assessment. A retrobulbar depot-injection of a corticosteroid should be considered in the protection of the optic nerve during treatment.

Improvement of central visual acuity outweighed impairment, but the difficulties involved in testing these handicapped patients, especially at the first examination, should not be forgotten. The two young patients who went blind during the trial would probably have done so without treatment, as we have seen that happen to similar cases in our longitudinal population survey (Anderson et al., 1976c).

\section{Conclusions}

The conclusions from this study, and from our experiences in the treatment of other heavily-infected patients with ocular onchocerciasis, are that it is possible to rid even the most heavily-infected patient of microfilariae without unacceptable side effects provided a correctly balanced and supervised DEC/ betamethasone regimen is used. However, more studies are necessary to find the optimal regimen of betamethasone dosage, as well as the optimal, i.e., effective and non-damaging, profile of DEC delivery in patients with different degrees of infection.

The initial DEC/betamethasone course made a subsequent 4-week suramin course tolerable, and the only serious adverse effect observed during suramin treatment of 54 persons was the aggravation of an iritis which, if left unmanaged, might have led to visual deficit. However, the development of retinal pigment epithelial atrophy and optic atrophy following this combined course calls for caution and further investigations. Detailed studies using fundus angiography during both DEC and suramin treatment are needed, as well as a better general understanding of the toxicity of both drugs.

We thank Professor Barrie Jones and Mr Alan Bird for helpful criticism of the manuscript. We acknowledge the help of our assistants in the field.

This study was supported by the Medical Research Council, London, and the World Health Organisation, Geneva.

\section{References}

Anderson, J., and Fuglsang, H. (1973). Variation in numbers of microfilariae of Onchocerca volvulus in the anterior chamber of the human eye. Transactions of the Royal Society of Tropical Medicine and Hygiene, 67, 544-548.

Anderson, J., and Fuglsang, H. (1977). Ocular onchocerciasis. Tropical Diseases Bulletin, 74, 257-272.

Anderson, J., Fuglsang, H., Hamilton, P. J. S., and Marshall, T. F. de C. (1974). Studies on onchocerciasis in the United Cameroon Republic. I. Comparison of populations with and without Onchocerca volvulus. Transactions of the Royal Society of Tropical Medicine and Hygiene, 68, 190208.

Anderson, J., Fuglsang, H., and Marshall, T. F. de C. (1976a). Effects of suramin on ocular onchocerciasis. Tropenmedizin und Parasitologie, 27, 279-296.

Anderson, J., Fuglsang, H., and Marshall, T. F. de C. (1976b). Effects of diethylcarbamazine on ocular onchocerciasis. Tropenmedizin und Parasitologie, 27, 263-278.

Anderson, J., Fuglsang, H., and Marshall, T. F. de C. (1976c). Studies on onchocerciasis in the United Cameroon Republic. III. A four year follow-up of 6 rain forest and 6 Sudan savanna villages. Transactions of the Royal Society of Tropical Medicine and Hygiene, 70, 362-373.

Bangham, D. R. (1955). Metabolism and excretion of ${ }^{14} \mathrm{C}$ labelled diethylcarbamazine. British Journal of Pharmacology, 10, 397-405.

Bartlett, A., Turk, J., Ngu, J., Mackenzie, C. D., Fuglsang, H., and Anderson, J. (1978). Variation in delayed hypersensitivity in onchocerciasis. (In press).

Bird, A. C., Andersson, J., and Fuglsang, H. (1976). Morphology of posterior segment lesions of the eye in patients with onchocerciasis. British Journal of Ophthalmology, 60, 2-20.

Bryceson, A. D. M. (1976). What happens when microfilariae die? Transactions of the Royal Society of Tropical Medicine and Hygiene, 70, 397-399.

Bryceson, A. D. M., Warrell, D. A., and Pope, H. M. (1977). Dangerous reactions to treatment of onchocerciasis with diethylcarbamazine. British Medical Journal, 1, 742-744.

Budden, F. H. (1959). Onchocerciasis therapy. Transactions of the Royal Society of Tropical Medicine and Hygiene, 53, 118-119.

Connor, D. H., Morrison, N. E., Kerdel-Vegas, F., Berkoff, H. A., Johnson, F., Tunnicliffe, R., Failing, F. C., Hale, L. N., and Lindquist, K. (1970). OnchocerciasisOnchocercal dermatitis, lymphadenitis, and elephantiasis in the Ubangi Territory. Human Pathology, 1, 553-579.

Duke, B. O. L. (1968a). The effects of drugs on Onchocerca volvulus. 3. Trials of suramin at different dosages and a 
comparison of the brands antrypol, moranyl, and naganol. Bulletin of the World Health Organisation, 39, 157-167.

Duke, B. O. L. (1968b). The effects of drugs on Onchocerca volvulus, 1 . Methods of assessment, population dynamics of the parasite and the effects of diethylcarbamazine. Bulletin of the World Health Organisation, 39, 137-146.

Duke, B. O. L., and Anderson, J. (1972). Onchocerciasis and its treatment. Tropical Doctor, 2, 107-114.

Fuglsang, H., and Anderson, J. (1974a). Collapse during treatment of onchocerciasis with diethylcarbamazine. Transactions of the Royal Society of Tropical Medicine and Hygiene, 68, 72-73.

Fuglsang, H., and Anderson, J. (1974b). Side effects of suramin. In Research and Control of Onchocerciasis in the Western Hemisphere. Pan American Health Organisation, Scientific Publication No. 298, pp. 54-56.

Fuglsang, H., and Anderson, J. (1977). The concentration of microfilariae in the skin near the eye as a simple measure of the severity of onchocerciasis in a community and as an indicator of danger to the eye. Tropenmedizin und Parasitologie, 28, 63-67.

Gibson, D. W., Duke, B. O. L., and Connor, D. H. (1977). Histopathological studies on suramin toxicity in a chimpanzee. Tropenmedizin und Parasitologie, 28, 387-405.

Hawking, F. (1940). Concentration of Bayer 205 (Germanin) in human blood and cerebrospinal fluid after treatment. Transactions of the Royal Society of Hygiene and Tropical Medicine, 34, 37-52.

Jones, B. R., Anderson, J., and Fuglsang, H. (1978). Effects of different concentrations of diethylcarbamazine drops in ocular onchocerciasis. British Journal of Ophthalmology, 62, 428-439.

Lubran, M. (1950). Determination of hetrazan in biological fluids. British Journal of Pharmacology, 5, 210-216.

Markell, E. K., and Turner, J. (1957). Cortisone and prednisone in the suppression of allergic reactions to diethylcarbamazine treatment of onchocerciasis. American Journal of Tropical Medicine and Hygiene, 15, 546-552.

Metge, P., Chovet, M., Cazenave, P., and Loréal, E. (1974). Lésions chorio-rétiniennes localisées dans l'onchocercose: Aspects angiofluorographiques. Médecine Tropicale, 34, 625-632.

Nelson, G. S. (1955). A preliminary report on the out-patient treatment of onchocerciasis with antrypol in the West Nile District of Uganda. East African Medical Journal, 32, 413-429.

Oomen, A. P. (1969). Fatalities after treatment of onchocerciasis with diethylcarbamazine. Transactions of the Royal Society of Tropical Medicine and Hygiene, 63, 548.

Satti, M. H., and Kirk, R. (1957). Observations on the chemotherapy of onchocerciasis in Bahr El Ghazal Province, Sudan. Bulletin of the World Health Organisation, 16, 531-540.

Trojan, H. (1975). Fundusveränderungen im Sinne einer 'flecked retina' bei Onchozerkose. Klinische Monatsblätter für Augenheilkunde, 166, 220-227.

World Health Organisation (1974). Onchocerciasis. Edited by A. A. Buck. Pp. 84. World Health Organisation: Geneva. 